\title{
A Influência do Traço de Personalidade Neuroticismo na Suscetibilidade às Falsas Memórias
}

\author{
Luciana Moreira de Ávila \\ Lilian Milnitsky Stein ${ }^{1}$ \\ Pontifícia Universidade Católica do Rio Grande do Sul
}

\begin{abstract}
RESUMO - As falsas memórias são lembranças de eventos que na realidade nunca ocorreram. Porém, a pergunta que surge é quais características e traços de personalidade poderiam influenciar na suscetibilidade a esse tipo de distorção de memória. A fim de investigar as diferenças individuais e falsas memórias, o presente estudo testou em 150 estudantes universitários o efeito do traço de personalidade neuroticismo (baseado no modelo dos Cinco Grandes Fatores) na suscetibilidade às falsas memórias. Para isso foram utilizados como instrumentos a Escala Fatorial de Ajustamento Emocional/Neuroticismo e a versão brasileira do procedimento das Listas de Palavras Associadas, contendo palavras de cunho neutro e emocional (positivo e negativo). Os resultados mostraram que pessoas com alto neuroticismo apresentaram maior número de falsas memórias e uma melhor lembrança para palavras de valência emocional negativa.
\end{abstract}

Palavras-chave: falsas memórias; neuroticismo; cinco grandes fatores; emoção; diferenças individuais.

\section{The Influence of the Neuroticism Personality Trait on the Susceptibility to False Memories}

\begin{abstract}
False memories are recollections of events that in fact never happened. However, the question is which characteristics and personality traits could affect the susceptibility to this kind of memory distortion. The present study tested 150 college students to investigate the effect of the neuroticism personality trait (based on the Five Factor Model) on the susceptibility to false memories. The Factorial Scale of Emotional Adjustment/Neuroticism and the brazilian version of the Lists of Associated Words procedure, containing words of neutral and emotional nature (positive and negative) were used to assess memory performance. Our findings showed that individuals with high levels of neuroticism presented more false memories and a better remembrance forwords with negative emotional valence.
\end{abstract}

Key words: false memories; neuroticism; five-factor model; emotion; individual differences.

Ao longo do tempo, a memória sempre exerceu um fascínio por sua complexidade. Porém, no século passado esse interesse pela memória estendeu-se também para suas falhas. É por meio da memória que cada ser humano pode lembrar de sua história pessoal, de fatos, de conceitos e é por meio dela que o processo de aprendizagem torna-se viável. Entretanto, a memória também tem seu lado obscuro e frágil. Pode-se esquecer de forma rápida ou gradual eventos importantes ou até mesmo distorcer o passado de forma surpreendente (Schacter, 2001). Uma das falhas de memória são as falsas memórias. As falsas memórias caracterizam-se pela lembrança de eventos que na realidade nunca ocorreram. As informações são armazenadas na memória e, mais tarde, são recordadas como se tivessem sido verdadeiramente vividas (Roediger \& McDermott, 2000).

Um dos modelos teóricos explicativos para a compreensão das falsas memórias é a Teoria do Traço Difuso (Brainerd \& Reyna, 2005). Segundo essa teoria, a memória não é um sistema unitário, mas sim constituída de múltiplos sistemas independentes, contendo representações literais e de essência (Neufeld \& Stein, 2001). Enquanto que a me-

1 Endereço: Pós-Graduação em Psicologia, PUCRS, Av. Ipiranga, 6681, prédio 11, sala 933, Porto Alegre, RS, Brasil 90619-900. E-mail:lilian@pucrs.br mória de essência armazena somente o significado do fato ocorrido, a memória literal contém em si as lembranças dos detalhes específicos sobre o evento (Brainerd, Stein \& Reyna, 1998). Assim, segundo a Teoria do Traço Difuso, as falsas memórias ocorrem em função da lembrança de informações acerca do sentido das experiências (memórias de essência). Na recordação dos eventos, aspectos específicos e detalhados (memórias literais) podem dar lugar aos aspectos e representações de essência das lembranças - mais gerais e amplas - devido, principalmente, à fragilidade dos traços literais.

Embora a vulnerabilidade da memória em apresentar distorções venha sendo estudada há algum tempo, recentes pesquisas têm apontado para uma nova área: o papel das diferenças individuais na suscetibilidade às falsas memórias. Segundo Loftus (1997), um número crescente de investigações mostra que, sob circunstâncias adequadas, falsas recordações podem ocorrer facilmente com alguns tipos de pessoas. A pergunta que surge, e que pouco tem sido abordada na literatura, é que tipos de indivíduos seriam particularmente suscetíveis ou resistentes às falsas memórias? Tendo em vista essa questão, o presente estudo teve como objetivo principal testar o efeito do nível do traço de personalidade neuroticismo e seus sub-fatores na suscetibilidade às falsas memórias. Como objetivo específico, buscou investigar o efeito da emoção na memória em função do traço de personalidade neuroticismo.

Os primeiros estudos analisando diferenças individuais, 
associando traços de personalidade à sugestibilidade e à suscetibilidade às falsas memórias, começaram a surgir no final da década de 1980 (Christiaansen, Ỏchalek \& Sweeney, 1984; Gudjonsson, 1983; 1988). Esses estudos fizeram com que pesquisadores deixassem de lado a visão de que distorções de memória seriam apenas erros na evocação das lembranças, passando a ver que essas distorções, ligadas a características de personalidade, tratavam-se de um fenômeno consistente. Para Loftus e Calvin (2001), responder às questões sobre as características individuais relacionadas às falsas memórias pode auxiliar no desenvolvimento de procedimentos para trabalhar a memória e suas distorções, e também evitar a utilização de técnicas, por parte de psicoterapeutas, não apropriadas para determinados indivíduos.

Peiffer e Trull (2000) investigaram a relação de três variáveis de personalidade - condescendência, auto-estima e afeto negativo - com a sugestibilidade e produção de falsas memórias. Um dos instrumentos utilizados para a investigação de falsas memórias foi a Lista de Palavras Associadas (Roediger \& McDermott, 1995). Peiffer e Trull não encontraram resultados significativos com relação à variável autoestima, mas concluíram que tanto o afeto negativo quanto a condescendência pode predizer uma maior tendência às falsas memórias.

No que concerne ao debate entre falsas memórias e diferenças individuais, a dissociação, medida pela escala Dissociative Experiences Scale (Carlson \& Putnam, 1993), parece já ter sido estabelecida como uma explicação amplamente aceita para as distorções de memória (Eisen \& Lynn, 2001; Hyman \& Billings, 1998; Porter, Birt, Yuille \& Lehman, 2000; Wilkinson \& Hyman, 1998; Winograd, Peluso \& Glover, 1998). Uma hipótese para a relação da dissociação com a produção de falsas memórias é a de que indivíduos com tendências dissociativas tendem a ser menos confiantes em suas recordações, fazendo com que se tornem mais vulneráveis às falsas informações (Eisen \& Lynn, 2001).

Embora escassos, os estudos na área de personalidade e falsas memórias podem auxiliar na compreensão do processo de formação das falsas memórias. Quas, Qin, Schaaf e Goodman (1997) já apontavam para o despertar do interesse dos pesquisadores em relacionar traços de personalidade baseados no modelo dos Cinco Grandes Fatores com a tendência em produzir falsas memórias.

O modelo dos Cinco Grandes Fatores, conhecido como "Big Five" e considerado como uma versão moderna da teoria do traço, busca compreender a essência da natureza humana de acordo com suas diferenças individuais (McCrae \& John, 1992). O modelo considera que a personalidade é constituída por cinco grandes traços: 1) Extroversão, 2) Socialização, 3) Escrupulosidade, 4) Neuroticismo e 5) Abertura para Experiência.

No presente estudo, escolheu-se o fator neuroticismo para a investigação de sua relação com a suscetibilidade às falsas memórias pelas características desse traço de personalidade, e por já existir um instrumento validado para a realidade brasileira para sua avaliação (Hutz \& Nunes, 2001). O traço de personalidade neuroticismo refere-se ao nível crônico de desajustamento e instabilidade emocional. As pessoas passam a experenciar padrões emocionais associados a um desconforto psicológico causado por aflições, angústias e sofrimentos (McCrae \& John, 1992). Altos índices de neuroticismo identificam indivíduos propensos a sofrimentos psicológicos e que podem apresentar níveis importantes de ansiedade, depressão, hostilidade, vulnerabilidade, autocrítica e impulsividade. O neuroticismo, em níveis mais altos, também inclui tendência a idéias dissociadas da realidade, baixa tolerância à frustração, afetos negativos, baixa capacidade de controle dos impulsos, baixa auto-estima e respostas de coping mal adaptadas (Nunes \& Hutz, 2002).

Hutz e Nunes (2001) identificaram quatro sub-fatores dentro da Escala do Neuroticismo, quais sejam: vulnerabilidade, desajustamento psicossocial, ansiedade e depressão. Vulnerabilidade refere-se a sintomas típicos de transtornos de personalidade dependente e de esquiva, além da intensidade que as pessoas vivenciam sofrimentos em decorrência da falta de aceitação dos outros para consigo. O sub-fator desajustamento psicossocial agrupa sintomas típicos de transtorno antisocial e borderline. Já o sub-fator ansiedade inclui características como instabilidade emocional, variações de humor e disposição, enquanto que o da depressão engloba sintomas de desesperança, ideação suicida e similares (Hutz \& Nunes, 2001).

Para investigar a associação das diferenças individuais com a suscetibilidade para a produção de falsas memórias, Porter e cols. (2000) utilizaram, pela primeira vez, o modelo dos Cinco Grandes Fatores. O estudo mostrou que indivíduos menos extrovertidos e com tendência à dissociação apresentavam um maior número de falsas memórias, existindo uma correlação positiva significativa entre o traço de personalidade neuroticismo e dissociação.

Já Gudjonsson (1983) investigou a suscetibilidade dos participantes à sugestão de falsas informações durante o interrogatório policial. Neste estudo, indivíduos com alto neuroticismo e alta ansiedade-traço eram mais sugestionáveis à falsa informação e, conseqüientemente, apresentavam um número maior de falsas memórias. Uma das explicações sugeridas estaria no fato de que pessoas com alto neuroticismo possuem, dentre outras características, uma baixa auto-estima, o que as impediria de confiarem em seus próprios julgamentos e respostas. Em um estudo posterior, Gudjonsson (1988) investigou o efeito das estratégias de coping na suscetibilidade às falsas informações, concluindo que a baixa capacidade em utilizar estas estratégias estava diretamente relacionada a uma maior tendência do indivíduo em aceitar sugestões de informações distorcidas.

Seguindo a linha de investigação de Porter, Gudjonsson e outros, o presente estudo se propõe a investigar a influência do traço de personalidade neuroticismo na sucetibilidade às falsas memórias.

\section{Método}

\section{Delineamento}

A fim de testar a principal hipótese de que indivíduos com traço de personalidade neuroticismo apresentam maior sucetibilidade às falsas memórias, utilizou-se um delineamento quase-experimental $3 \times 2$, com a variável neuroticismo (baixo, padrão, alto) tendo sido tratada entre-grupos e a variável emoção (negativa, neutra e positiva) manipulada intra-grupos. Como variável dependente foi considerado o desempenho na memória (em termos de memórias verdadeiras e falsas).

\section{Participantes}

Participaram do estudo 150 alunos de graduação dos cursos de Administração de Empresas, Medicina Veterinária, Psicologia e Serviço Social de duas universidades do interior do Rio Grande do Sul, sendo uma particular e uma federal. A idade dos participantes variou entre 17 e 52 anos $(M=24,67$, $D P=7,97)$, sendo $117(78 \%)$ do sexo feminino e $33(22 \%)$ do sexo masculino. Os participantes foram escolhidos por conveniência e sua participação no estudo foi voluntária. 


\section{Instrumentos}

\section{Escala Fatorial de Ajustamento Emocional/ Neuroticismo - EFN}

A EFN é um instrumento auto-administrável, baseado no modelo dos Cinco Grandes Fatores, que permite uma avaliação rápida e objetiva de uma dimensão da personalidade humana denominada Neuroticismo/Estabilidade Emocional. A escala é composta por 82 itens em quatro sub-escalas: Vulnerabilidade, Desajustamento Psicossocial, Ansiedade e Depressão. Os itens avaliam quão adequadamente cada sentença aplica-se aos indivíduos e foram construídos na forma de frases que descrevem atitudes, crenças e sentimentos dos participantes. Por exemplo: "Freqüentemente sinto que coisas muito ruins estão por acontecer, mesmo sem nenhum motivo aparente". Os indivíduos fornecem suas respostas numa escala tipo Likert de sete pontos $(1$ - completamente inadequada, "a sentença não descreve nenhuma característica minha"; 4 - neutro, "mais ou menos"; e 7 - perfeitamente adequada, "a sentença me descreve perfeitamente bem"). A avaliação do teste resulta em escores percentílicos nas quatro dimensões do neuroticismo. A escala geral deve ser interpretada a partir de sua conversão em um escore padronizado. $\mathrm{O}$ instrumento apresenta boa consistência interna $($ alpha $=0,94)$ e boa capacidade de discriminação, servindo como um recurso útil para a indicação de transtornos de personalidade (Hutz \& Nunes, 2001).

\section{Procedimento da Lista de Palavras Associadas}

O Procedimento da Lista de Palavras Associadas é composto por um material original que contém 44 listas, cada uma com 15 palavras, denominadas alvos, girando em torno de um mesmo tema. Essas listas podem ser neutras ou possuir conteúdo emocional. Uma palavra crítica, ou seja, uma palavra que traduz a essência temática de cada lista não é apresentada durante a etapa de memorização das palavras. Por exemplo, todas as palavras da lista pátria, símbolo, nação, mastro, país, verde, pano, hino, flâmula, honra, identificação, representação, amarelo, haste e estado versam sobre o tema bandeira. Neste exemplo, bandeira não é incluída na lista de palavras-alvo, somente sendo incluída como um distrator crítico no teste de memória de reconhecimento.

Considerando que as Listas de Palavras Associadas, em sua versão brasileira de Stein, Feix \& Rohenkohl (2006), são compostas por 44 listas (35 listas "neutras" e nove listas "emocionais" - cinco listas de conteúdo emocional negativo e quatro listas de conteúdo emocional positivo), foi necessário estabelecer-se critérios para determinar quais delas fariam parte do material original a ser apresentado aos participantes do presente estudo. Foi estabelecido que fariam parte do estudo 12 listas de palavras - seis neutras e seis emocionais (três com conteúdo emocional negativo e três com conteúdo emocional positivo). Como critério para a escolha das listas de palavras utilizou-se a avaliação da valência emocional de cada lista, feita por juizes no estudo de Stein e cols. Para as listas ditas neutras foram escolhidas as que obtiveram um índice de emocionalidade mais próximo possível a zero. Enquanto que para as listas emocionais foram escolhidas aquelas com índices de emocionalidade mais altos (seja negativa ou positiva).

As 180 palavras das 12 listas selcionadas foram gravadas em áudio com equipamentos profissionais por uma pessoa do sexo feminino, com experiência em locução. $\mathrm{O}$ intervalo entre cada palavra foi de dois segundos, não ha- vendo separação entre as diferentes listas, sendo o tempo total de gravação de nove minutos. Com a intenção de controlar possíveis efeitos da ordem de apresentação das listas no material original, foram gravados dois CDs, diferenciados apenas pela diferente ordem aleatória de apresentação das listas (CD 1 e CD 2).

\section{Teste de Memória de Reconhecimento}

O teste de reconhecimento foi composto de 72 palavras, sendo estas divididas em três tipos de itens: 36 alvos (palavras estudadas no material original, sendo três palavras escolhidas aleatoriamente entre as posições 3-12 de suas respectivas listas), 12 distratores críticos (palavras não apresentadas no material original e que traduziam a essência semântica de cada uma das listas) e 24 distratores não relacionados (palavras não apresentadas no material original e sem relação semântica com o mesmo, selecionados do Estudo II de Stein \& cols., 2006). A ordem de disposição dos itens no teste de reconhecimento foi aleatória, respeitando a restrição de que alvos e distratores críticos da mesma lista não estivessem em sequiência imediata.

\section{Procedimento}

Os participantes foram testados em sala de aula, em grupo, e em uma única sessão. Inicialmente foram feitos a apresentação da pesquisa e os procedimentos éticos. Aqueles que concordaram em fazer parte do estudo assinaram o termo de consentimento livre e esclarecido.

Foi então solicitado aos participantes que escutassem com atenção às listas de palavras que iriam ser apresentadas, pois sua memória para essas listas seria testada posteriormente. Foram instruídos a não escrever nada enquanto escutavam as listas. A escolha de qual CD (CD 1 e CD 2) seria apresentado a cada grupo de alunos foi aleatória.

Ao final da apresentação das listas foi realizada uma tarefa de isolamento com o objetivo de impedir que as informações sobre as listas alvos mantivessem-se on line na memória. Essa tarefa, um conjunto de séries numéricas, teve duração de três minutos. Após, deu-se início ao teste de reconhecimento. Os participantes foram instruídos, em primeiro lugar, a preencher os dados referentes à idade, sexo e curso. Foi explicado que a folha que iriam receber continha várias palavras, sendo que algumas delas haviam sido apresentadas nas listas ouvidas anteriormente, enquanto outras eram palavras novas, não escutadas na apresentação em áudio. Eles deveriam circular somente aquelas palavras que tivessem ouvido exatamente igual na gravação.

Ao final, aplicou-se a Escala Fatorial de Ajustamento Emocional/Neuroticismo. As instruções da escala foram dadas de acordo com o manual de aplicação do instrumento. O tempo total despendido para a realização do experimento foi de, aproximadamente, 45 minutos.

\section{Análise dos dados}

Foram realizadas três ANOVAs, adotando-se em todas elas um nível de significância de $\alpha<0,05$. A primeira ANOVA investigou possíveis efeitos da ordem de apresentação do material aos participantes (CD1 e CD2) e da variável sexo (masculino e feminino); a segunda investigou o efeito do traço neuroticismo e seus sub-fatores na memória; a terceira avaliou o efeito da emoção na memória em função do traço de personalidade neuroticismo e seus sub-fatores. Testes pos hoc com correção para Bonferroni foram utiliza- 
dos para as comparações múltiplas significativas.

\section{Resultados}

A apresentação dos resultados foi feita tendo em vista o principal objetivo do presente estudo, qual seja, o de investigar o efeito do nível do traço de personalidade neuroticismo na suscetibilidade às falsas memórias. Segundo Hutz e Nunes (2001), escores padrão entre 80 e 120 são esperados para a maior parte da população e, portanto, foram utilizados no presente estudo para definição do nível padrão de neuroticismo. Escores abaixo de 80 foram considerados como nível baixo e acima de 120 como nível alto. Os participantes, nos três diferentes níveis do traço neuroticismo total, ficaram assim distribuídos: 11 participantes no nível baixo $(M=77,7 ; D P=1,63), 123$ pessoas no nível padrão $(M=97,85 ; D P=1,92)$ e 16 indivíduos no nível alto $(M=127,17 ; D P=4,81)$.

Para a apresentação dos índices de respostas baseadas na memória, no que tange as memórias verdadeiras, foram utilizadas as médias de reconhecimento (responder "sim") aos itens-alvo, itens estes que foram apresentados no material original (por exemplo, mastro). Para as falsas memórias, utilizou-se a média de aceitação, no teste de reconhecimento, dos distratores críticos (palavras não apresentadas no material original e que resumiam a essência semântica das listas, por exemplo, bandeira).

Em primeiro lugar, investigou-se o possível efeito da ordem de apresentação do material original (CD 1 e CD 2) no desempenho dos participantes, não evidenciandose efeito principal da ordem do material $[F(1,148)=2,16$, $p>0,05]$, ou interações dessa variável com tipo de memória $[F(1,148)=1,07, p>0,05]$.

Com relação a possível interferência da variável sexo, não foram encontradas diferenças estatisticamente sig-

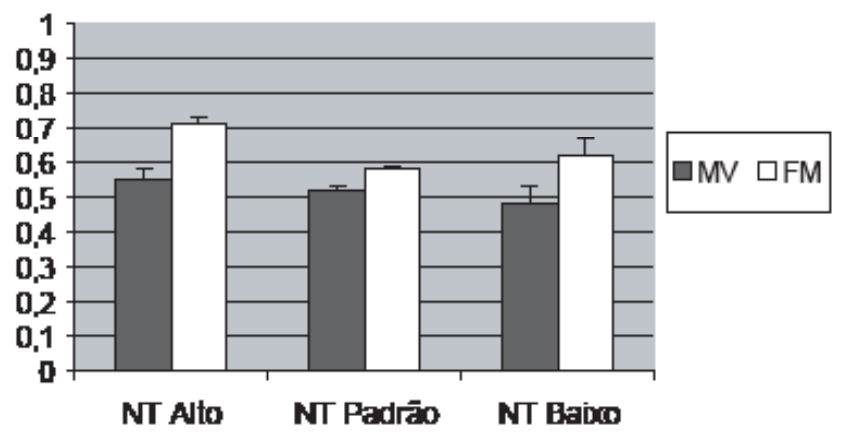

Figura 1. Média de memórias verdadeiras (MV) e de falsas memórias (FM) em função dos níveis de neuroticismo total (NT).

nificativas no desempenho da memória quando comparados o grupo de participantes homens com o de mulheres $[F(2,147)=1,49, p>0,05]$, mesmo quando considerada a variável emoção $[F(3,218)=1,23, p>0,05]$. Portanto, as variáveis relativas à ordem de apresentação do material original e ao sexo serão desconsideradas nas próximas análises.

Quanto ao desempenho geral no teste de memória, observou-se que, de uma maneira geral, os participantes apresentaram uma média significativamente maior de falsas memórias $(M=0,6, D P=0,16)$ do que de memórias verdadeiras $(M=0,52, D P=0,13)[t(149)=-5,55, p<0,001]$, o que pode ser esperado, quando o procedimento de Listas de Palavras Associadas emprega um teste de reconhecimento (Roediger \& McDermott, 1995). Em relação aos distratores não relacionados, ou seja, respostas dadas sem base mnemônica, observou-se uma média de respostas para esse tipo de item praticamente insignificante $(M=0,02, D P=0,05)$, indicando que os participantes apresentaram uma boa qualidade de memória no teste de reconhecimento. Sendo assim, os índices de distratores não relacionados não foram incluídos nas

Tabela 1. Média de Memórias Verdadeiras e Falsas para Palavras com Valência Emocional Positiva, Neutra e Negativa (Desvio Padrão entre Parênteses).

\begin{tabular}{|c|c|c|c|c|c|c|}
\hline \multirow[b]{3}{*}{ Vulnerabilidade } & \multicolumn{3}{|c|}{ Memórias Verdadeiras } & \multicolumn{3}{|c|}{ Memórias Falsas } \\
\hline & + & neutra & - & + & neutra & - \\
\hline & & & & & & \\
\hline Alto & $.55(.21)$ & $.45(.17)$ & $.70(.25)^{\mathrm{a} b}$ & $.53(.32)$ & $.67(.20)^{\mathrm{b}}$ & $.77(.31)$ \\
\hline Padrão & $.62(.19)$ & $.49(.21)$ & $.55(.28)^{\mathrm{a}}$ & $.58(.30)$ & $.57(.25)$ & $.62(.33)$ \\
\hline Baixo & $.56(.23)$ & $.41(.20)$ & $.55(.27)^{\mathrm{b}}$ & $.64(.28)$ & $.48(.24)^{\mathrm{b}}$ & $.65(.32)$ \\
\hline \multicolumn{7}{|c|}{ Desajustamento Psicossocial } \\
\hline Alto & $.56(.18)$ & $.43(.17)$ & $.61(.29)$ & $.51(.33)$ & $.64(.21)^{\mathrm{b}}$ & $.69(.34)$ \\
\hline Padrão & $.61(.22)$ & $.44(.20)$ & $.56(.27)$ & $.61(.28)$ & $.53(.27)$ & $.71(.31)$ \\
\hline Baixo & $.57(.22)$ & $.49(.22)$ & $.60(.28)$ & $.62(.29)$ & $.53(.23)^{\mathrm{b}}$ & $.62(.33)$ \\
\hline \multicolumn{7}{|c|}{ Ansiedade } \\
\hline Alto & $.57(.19)$ & $.44(.20)$ & $.67(.26)^{\mathrm{a}}$ & $.56(.31)$ & $.58(.23)$ & $.75(.31)$ \\
\hline Padrão & $.61(.20)$ & $.44(.21)$ & $.54(.26)^{\mathrm{a}}$ & $.59(.30)$ & $.53(.26)$ & $.64(.35)$ \\
\hline Baixo & $.56(.24)$ & $.48(.20)$ & $.56(.29)$ & $.61(.30)$ & $.58(.25)$ & $.64(.32)$ \\
\hline \multicolumn{7}{|c|}{ Depressãa } \\
\hline Alto & $.54(.22)$ & $.42(.19)$ & $.72(.26)^{\mathrm{a} b}$ & $.53(.33)$ & $.61(.25)$ & $.77(.31)$ \\
\hline Padrão & $.61(.19)$ & $.49(.19)$ & $.54(.28)^{\mathrm{a}}$ & $.63(.29)$ & $.55(.23)$ & $.61(.34)$ \\
\hline Baixo & $.58(.21)$ & $.44(.21)$ & $.54(.26)^{\mathrm{b}}$ & $.59(.29)$ & $.54(.25)$ & $.65(.32)$ \\
\hline \multicolumn{7}{|c|}{ Neuroticismo Total } \\
\hline Alto & $.51(.24)$ & $.43(.16)$ & $.84(.19)^{\mathrm{a} b}$ & $.50(.38)$ & $.71(.17)^{\mathrm{a}}$ & $.96(.11)^{\mathrm{a} b}$ \\
\hline Padrão & $.59(.20)$ & $.46(.21)$ & $.56(.27)^{\mathrm{a}}$ & $.58(.29)$ & $.55(.25)^{\mathrm{a}}$ & $.64(.33)^{\mathrm{a}}$ \\
\hline Baixo & $.52(.27)$ & $.43(.19)$ & $.53(.31)^{\mathrm{b}}$ & $.70(.28)$ & $.54(.24)$ & $.70(.35)^{\mathrm{b}}$ \\
\hline
\end{tabular}

${ }^{\text {a }}$ Diferenças significativas entre grupos de nível alto e padrão.

${ }^{\mathrm{b}}$ Diferenças significativas entre grupos de nível alto e baixo.

$p<.05$. 
próximas análises.

\section{Neuroticismo Total e Memória}

Para investigar o efeito do neuroticismo total na memória, conduziu-se uma ANOVA 3 (nível de neuroticismo total: baixo, padrão e alto) x 2 (tipo de memória: verdadeira e falsa), com medidas repetidas para a última variável. Não foi encontrado um efeito principal para nível de neuroticismo total $[F(2,147)=2,05, p>0,05]$. Entretanto, evidenciou-se um efeito principal para tipo de memória $[F(1,147)=29,19, p<0,01]$, em função da supremacia das falsas memórias sobre as memórias verdadeiras, como já mencionado. Esse efeito foi qualificado por uma interação, apresentada na Figura 1, entre nível de neuroticismo e tipo de memória (verdadeira e falsa) $[F(2,147)=3,51, p<.05]$. Testes post hoc entre grupos mostraram que indivíduos com alto neuroticismo apresentaram uma média de falsas memórias significativamente maior $(M=0,72, D P=0,10)$ do que participantes com neuroticismo padrão $(M=0,58, D P=0,20)$ $[t(137)=2,62, p<0,05]$. Não foram verificadas diferenças significativas entre as médias de falsas memórias nas outras comparações entre os grupos de neuroticismo, assim como naquelas para memórias verdadeiras ( $p s>0,05)$.

A análise de variância revelou uma interação entre valência emocional, tipo de memória e nível de neuroticismo $[F(4,294)=2,36, p<0,05]$. Como pode ser observado nas três últimas linhas da Tabela 1, indivíduos com alto neuroticismo apresentaram taxas mais elevadas de memórias verdadeiras e falsas para palavras de conteúdo emocional negativo, tanto na comparação com o grupo com neuroticismo padrão [ts $(137)>3,88, p s<0,01]$, quanto com o grupo com nível baixo de neuroticismo [ts(25)>-2,81, $p s<0,05]$. Ainda, o índice de falsas memórias para palavras neutras também foi maior para indivíduos com alto neuroticismo do que para indivíduos com neuroticismo padrão [ $t(137)=2,48, p<0,05]$. Por outro lado, mesmo levando-se em conta a valência emocional do material, não foram evidenciadas diferenças significativas entre os grupos com níveis de neuroticismo baixo e padrão [ts(132)>0,42, ps>0,05]. Já o desempenho da memória para palavras com conteúdo emocional positivo não apresentou diferenças significativas na comparação entre os grupos de neuroticismo ( $p s>0,05)$.

\section{Fatores do Neuroticismo e a Memória}

Nesta etapa foram analisados os possíveis efeitos, na memória, dos traços de personalidade que compõem o escore do neuroticismo total - vulnerabilidade, desajustamento psicossocial, ansiedade e depressão (ver Tabela 1).

Nenhum dos sub-fatores do neuroticismo apresentaram efeito principal no desempenho da memória $[F s(2,147)>0,31$, $p s>0,05]$. Também não houve interações entre tipo de memória e o nível dos sub-fatores $[F s(2,147)>0,23, p s>0,05]$. Entretanto, em relação à vulnerabilidade, houve uma tendência, marginalmente significativa $(p=0,58)$ dos índices de falsas memórias apresentarem-se maiores $(M=0,65, D P=0,17) \mathrm{em}$ participantes com alto nível de vulnerabilidade do que com pessoas com baixo nível deste traço $(M=0,56, D P=0,20)$.

Quando analisados os possíveis efeitos da emoção na memória em função do nível dos sub-fatores, somente para os de vulnerabilidade e desajustamento psicossocial foram verificadas interações significativas $[F s(4,294)>4,7$, $p s<0,01]$. Quanto à vulnerabilidade (três primeiras linhas da Tabela 1), a testagem post hoc evidenciou que pessoas com alto nível desse traço apresentaram uma média de memórias verdadeiras para palavras negativas significativa- mente maior do que aquelas com vulnerabilidade padrão [t(98) $2,73, p<0,05]$ e baixa $[t(91)=-2,73, p<0,05]$. Também foi encontrada uma diferença significativa entre a média de falsas memórias para palavras neutras $[t(91)=-4,03, p<0,05]$ entre os grupos com baixa e alta vulnerabilidade. Em relação às outras médias do traço de personalidade vulnerabilidade não foram encontradas diferenças estatisticamente significativas $(p s>0,05)$.

Em relação ao sub-fator desajustamento psicossocial, as análises das interações mostraram que participantes com alto nível desse fator apresentaram maior número de falsas memórias para palavras neutras do que pessoas com nível baixo do traço $[t(96)=-2,49, \mathrm{p}<0,05]$ (linhas 4 e 6 , coluna 5 da Tabela 1).

No que concerne os sub-fatores ansiedade e depressão, as interações estatisticamente significativas concentraramse na relação entre emoção e nível dos sub-fatores do neuroticismo $[F s(4,294)>2,54, p s<0,05]$. Em relação ao primeiro desses sub-fatores, pode-se observar (linhas 7 e 8, coluna 3 da Tabela 1) que indivíduos com nível alto de ansiedade apresentaram uma média maior de memórias verdadeiras para palavras de cunho emocional negativo do que pessoas com ansiedade num nível padrão [ $t(101)=2,55, p<0,05]$.

Já na depressão, notou-se o efeito da emoção negativa do material no desempenho das pessoas com alto nível de depressão (linhas 1, 11 e 12, colunas 3 e 6 da Tabela 1). Estas apresentaram média superior de memórias verdadeiras e falsas quando comparadas com as pessoas com nível padrão de depressão $[t s(90) 2,42, p s<0,05]$, bem como apresentaram maior número de memórias verdadeiras para palavras negativas do que indivíduos com baixo nível de depressão $[t(99)=-3,44, p<0,05]$

\section{Discussão}

Os dados sugerem que indivíduos com níveis de neuroticismo diferentes daquele do nível padrão, esperado para a população, apresentam mais falsas memórias, corroborando a principal hipótese de que o traço de personalidade neuroticismo parece estar relacionado a uma maior suscetibilidade às falsas memórias.

A dificuldade de interpretar esse resultado advém do fato de que a literatura específica nesta área ainda é escassa. Porém, tendo em vista que pessoas com alto neuroticismo apresentam como características, altos níveis de instabilidade emocional, sofrimento psicológico, ansiedade, depressão, vulnerabilidade e autocrítica (Costa \& Widiger, 1993), torna-se viável estabelecer possíveis relações que expliquem a influência do neuroticismo no desempenho da memória. A primeira direção que pode ser apontada encontra-se no fato de que pessoas com alto neuroticismo tendem a ter altos níveis de vulnerabilidade, afetos negativos, baixa auto-estima e respostas de coping mal adaptadas (McCrae \& Costa, 1997) - características que podem estar associadas à suscetibilidade às falsas memórias (Gusjonsson, 1983, 1988; Peiffer \& Trull, 2000)

Neste sentido, os resultados obtidos no estudo de Porter e cols. (2000), que foi um dos pioneiros a investigar a possível influência dos cinco traços de personalidade, dentro do modelo dos Cinco Grandes Fatores, nas distorções de memória, evidenciaram que o fator neuroticismo apresentou uma correlação com dissociação, característica amplamente associada à suscetibilidade às falsas memórias (Eisen \& Lynn, 2001; Hyman \& Billings, 1998; Porter \& cols. 2000; Wilkinson \& Hyman, 1998; Winograd \& cols., 1998). Essa relação poderia ser um fator determinante na suscetibilida- 
de às falsas memórias, já que pessoas com alto neuroticismo tendem a apresentar idéias dissociadas da realidade (Hutz \& Nunes, 2001).

Outro conceito, que também pode estar contribuindo para que diferenças de personalidade possam afetar a produção de falsas memórias, diz respeito ao fato de que pessoas com alto traço de neuroticismo trazem consigo uma baixa capacidade de utilizarem estratégias de coping (Nunes \& Hutz, 2002). Segundo o modelo sugerido por Gudjonsson (1988), pessoas com baixas estratégias de coping - por exemplo, em um interrogatório - não conseguem estabelecer uma avaliação crítica das questões que lhes são apresentadas e acabam desenvolvendo uma tendência a fornecer respostas afirmativas a perguntas, gerando, assim, maior número de distorções de memória.

Observou-se também que indivíduos com alta vulnerabilidade apresentaram maior tendência, embora marginalmente significativa, às falsas memórias. Esse dado vai ao encontro dos estudos de Gudjonsson $(1983,1988)$ que mostraram que pessoas, cuja memória tende a ser mais sugestionável, possuem uma necessidade de reduzir a sensação de incerteza, apresentam menos confiança em seus próprios julgamentos e possuem maior medo de uma avaliação negativa por parte de outras pessoas. Estas são características da personalidade de esquiva presente em indivíduos com altos escores em vulnerabilidade.

Quanto ao efeito da emocionalidade na memória, observa-se, tanto no neuroticismo total quanto em seus sub-fatores, uma forte tendência para o reconhecimento de material com cunho emocional negativo, sejam elas memórias verdadeiras ou falsas. Martin, Ward e Clarck (1983) também mostraram uma tendência nesta direção, já que pessoas com altos escores em neuroticismo recordavam de palavras negativas sobre si mesmos em um número significativamente maior do que indivíduos com baixos escores. Sabe-se que pessoas com alto neuroticismo tendem a uma supervalorização de fatos negativos, além de interpretarem estímulos ambíguos de forma negativa ou ameaçadora (Larsen, 1992; Watson \& Hubbard, 1996).

Segundo o estudo de Larsen (1992), o fator neuroticismo encontra-se associado a elevados níveis de auto-relatos de doenças. Ou seja, pessoas com alto traço de neuroticismo tendem a exagerar em seus relatos sobre a vivência de prejuízos na saúde. Para Larsen, isso ocorre porque as pessoas com alto neuroticismo são mais ruminativas, apreensivas e pessimistas. Lishman (1972) já apontava para o fato de que indivíduos com alto neuroticismo têm maior tendência para recordar informações com tons desagradáveis. Essa tendência parece ocorrer na codificação seletiva de informações negativas que acaba por criar as condições necessárias para a recordação também seletiva dos conteúdos negativos (Larsen, 1992).

Seguindo a análise dos resultados obtidos no estudo de Larsen (1992), pode-se pensar que, como existe uma tendência de pessoas com alto neuroticismo em apresentar seus relatos de doenças de forma mais exagerada, esses relatos também carregariam, potencialmente, distorções de memória. Essas distorções poderiam advir justamente da codificação e recordação seletiva destes indivíduos. Essas lembranças seletivas abririam espaços para vieses de memória, conseqüientes de reconstruções de experiências vividas no passado (por exemplo, o relato exagerado de doenças).

Algumas limitações do presente estudo podem ser explicitadas para que sejam aperfeiçoadas e melhor investigadas futuramente. Uma limitação metodológica diz respeito ao instrumento utilizado. O procedimento das Listas de Palavras Associadas, desenvolvido para o Brasil por Stein e cols. (2006), apresenta-se como um recurso poderoso na produção de falsas memórias e também proporciona um bom controle experimental. Entretanto, o instrumento mede memórias semânticas. O sistema de memória semântica pode ser definido como aquele responsável pelo armazenamento de conhecimentos gerais que o indivíduo possui sobre o mundo, tais como conhecimentos de fatos, conceitos e vocabulário (Izquierdo, 2002). Em estudos futuros, sugerem-se outros tipos de investigação e procedimentos para testar a capacidade dos resultados serem replicados em diferentes tipos de memória, como, por exemplo, com memórias autobiográficas, que embora perca-se em rigor experimental, oferecem a vantagem de uma maior validade ecológica.

A título de síntese, pode-se concluir que o traço de personalidade neuroticismo apresenta um efeito significativo na suscetibilidade às falsas memórias. Embora o traço vulnerabilidade também tenha aparecido como um preditor às falsas memórias, observou-se que o efeito do neuroticismo na memória é potencializado quando níveis elevados coexistem em todas as sub-escalas do instrumento. Esse resultado vai ao encontro do estudo de Kizilbash, Vanderploeg e Curtiss. (2002) que, analisando os efeitos de ansiedade e depressão na memória, só encontraram resultados significativos quando os dois traços apareciam concomitantemente.

$\mathrm{O}$ presente estudo possui um caráter inovador. $\mathrm{O}$ fato de ser o primeiro no Brasil a investigar diferenças individuais nas falsas memórias, busca de forma pioneira contribuir para uma área promissora e ainda pouco explorada. Para Hyman e Billings (1998), as diferenças individuais estão certamente relacionadas à produção de falsas memórias, embora ainda esteja pouco claro como essas diferenças contribuem para as distorções de memória. Clarificar esse campo pode contribuir de forma fundamental para áreas como a Psicologia Clínica e Jurídica. Na área clínica, o conhecimento da influência do traço de personalidade na suscetibilidade às falsas memórias ajudaria os terapeutas no uso de técnicas para recuperação de memórias adequadas para cada tipo de paciente (Kihlstrom, 1981). Outra contribuição encontra-se na área jurídica, campo no qual as memórias trazidas pelas testemunhas poderiam ser interpretadas de acordo com as características individuais de cada uma delas, auxiliando no grau de confiança depositado em um relato testemunhal e no desenvolvimento de técnicas mais adequadas no sentido de otimizar a qualidade do testemunho.

\section{Referências}

Brainerd, C. J. \& Reyna, V. F. (2005). The science offalse memory. New York: Oxford University Press.

Brainerd, C. J., Stein, L. M. \& Reyna, V. F. (1998). On the development of conscious and uncounscious memory. Development Psychology, 34, 342-357.

Carlson, E. B. \& Putnam, F. W. (1993). An update on the Dissociative Experiences Scale (DES). Dissociation Progress on the Dissociative Disorders, 6, 16-27.

Christiaansen, R. E., Ochalek, K. \& Sweeney, J. D. (1984). Individual differences in eyewitness memory and confidence judgments. The Journal of General Psychology, 11, 47-52.

Costa, P. T., Jr. \& Widiger, T. A. (1993). Introduction. Em P. T. Costa \& T. A. Widiger, (Orgs.), Personality Disorders and the Five Factor Model of Personality (pp. 1-10), Washington, DC: American Psychological Association.

Eisen, M. L. \& Lynn, S. J. (2001). Dissociation, memory and suggestibility in adults and children. Applied Cognitive Psychology, $15,49-73$. 
Gudjonsson, G. H. (1983). Suggestibility, intelligence, memory recall and personality: An experimental study. British Journal Psychiatric, 142, 35-37.

Gudjonsson, G. H. (1988). Interrogative suggestibility: Its relationship with assertiveness, social-evaluative anxiety, state anxiety and method of coping. British Journal of Clinical Psychology, 27, 159-166.

Hutz, C. S. \& Nunes, C. H. (2001). Escala fatorial de ajustamento emocional/ Neuroticismo - EFN. São Paulo: Casa do Psicólogo.

Hyman, I. E. \& Billings, F. J. (1998). Individual differences and the creation of false childhood memories. Memory, 6(1), 1-20.

Izquierdo, I. (2002). Memória. Porto Alegre: Artmed.

Kihlstrom, J. F. (1981). Personality and memory. Em N. Cantor \& J.F. Kihlstrom (Orgs.), Personality, Cognition, and Social Interaction (pp.123-149). Hillsdale: Lawrence Erlbaum.

Kizilbash, A. H., Vanderploeg, R. D., \& Curtiss, G. (2002). The effects of depression and anxiety on memory performance. Archives of Clinical Neuropsychology, 17, 57-67.

Larsen, R. J. (1992). Neuroticism and selective encoding and recall of symptoms: Evidence from a combined concurrent-retrospective study. Journal of Personality and Social Psychology, 62(3), 480-488.

Lishman, W. A. (1972). Selective factor in memory. Part 1: Age, sex, and personality attributes. Psychological Medicine, 2, 121-138.

Loftus, E. F. (1997). Creating false memories. Scientific American, 277, 70-75.

Loftus, E. F., \& Calvin, W. (2001). Memory's future. Psychology Today, 34(2), 55-58.

Martin, M., Ward, J. C. \& Clarck, D. M. (1983). Neuroticism and the recall of positive and negative personality information. Behavior Research and Therapy, 21, 495-503.

McCrae, R. R. \& Costa, P. T. (1997). Personality trait structure as a human universal. American Psychologist, 52, 509-516.

McCrae, R. R. \& John, O. P. (1992). An introduction to the FiveFactor Model and its applications. Journal of Personality, 6, 175-216.

Neufeld, C. B., \& Stein, L. M. (2001). A compreensão da memória segundo diferentes perspectivas teóricas. Estudos de Psicologia, 18(2), 50-63.

Nunes, C. H. S. S. \& Hutz, C. S. (2002). O modelo dos Cinco Grandes Fatores de personalidade. Em R. Primi (Org.), Temas em Avaliação Psicológica (pp. 40-49). Campinas: IBAP.

Peiffer, L. C. \& Trull, T. J. (2000). Predictors of suggestibility and false memory production in young adult women. Journal of Personality Assessment, 74(3), 384-399.
Porter, S., Birt, A. R., Yuille, J. C. \& Lehman, D. R. (2000). Negotiating false memories: Interviewer and rememberer characteristics relate to memory distortion. Psychological Science, 11(6), 507-510.

Quas, J. A., Qin, J., Schaaf, J. M. \& Goodman, G. S. (1997). Individual differences in children's and adults' suggestibility and false event memory. Learning and Individual Differences, 9(4), 359-390.

Roediger, H. L. III. \& McDermott, K. B. (1995). Creating false memories: Remembering words not presented on lists. Journal of Experimental Psychology: Learning, Memory, and Cognition, 21, 803-814.

Roediger, H. L. III. \& McDermott, K. B. (2000). Distortions of memory. Em E. Tulving \& F. I. M. Craik (Orgs.), The Oxford Handbook of Memory (pp. 149-162). Oxford: Oxford University Press.

Schacter, D. L. (2001). The seven sins of memory: How the mind forgets and remembers. New York: Houghton Mifflin Company.

Stein, L. M., Feix, L. \& Rohenkohl, G. (2006). Avanços metodológicos no estudo das falsas memórias: Construção e normatização do procedimento de palavras associadas à realidade brasileira. Psicologia: Reflexão e Crítica, 19(2), pp. 196-205.

Watson, D. \& Hubbard, B. (1996). Adaptational style and dispositional structure: Coping in the context of the five-factor model. Journal of Personality, 64, 737-774.

Wilkinson, C. \& Hyman, I. E. (1998). Individual differences related to two types of memory errors: Word lists may not generalize to autobiographical memory. Applied Cognitive Psychology, 12, 29-46.

Winograd, E., Peluso, J. P. \& Glover, T. A. (1998). Individual differences in susceptibility to memory illusions. Applied Cognitive Psychology, 12, 5-28.s 


\section{CONGRESSO NORTE NORDESTE DE PSICOLOGIA}

(V CONPSI)

Produção e Diversidade: Compromissos Éticos e Políticos em Psicologia

Data: 23 a 26 de Maio de 2007

Local: Centro Cultural e de Exposições de Maceió - Maceió/AL

\section{Organização}

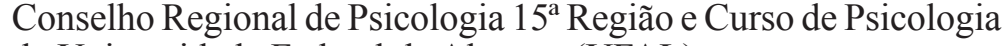
da Universidade Federal de Alagoas (UFAL)

Informações e inscrições

http://www.crp15.org.br/vconpsi

e-mail:vconpsi-secretaria@crp15.org.br 